\title{
Le point sur la conciliation entre la vie professionnelle et la vie privée dans le droit communautaire
}

\section{Reconciliation of work and private life in EU law: state of affairs}

\section{Susanne Burri}

Published online: 18 February 2010

(C) The Author(s) 2010. This article is published with open access at Springerlink.com

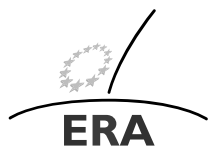

EUROPÄISCHE RECHTSAKADEMIE ACADEMY OF EUROPEAN LAW ACADEMIE DE DROIT EUROPEEN ACCADEMIA DI DIRITTO EUROPEO TRIER - TREVES - TREVIRI

Résumé Cet article offre un aperçu des objectifs de l'Union européenne, des développements législatifs actuels au niveau communautaire et de la jurisprudence de la Cour de Justice de l'Union européenne en matière de conciliation entre vie professionnelle, vie privée et vie familiale. Alors que le taux d'emploi des femmes atteindra en moyenne probablement l'objectif fixé dans le cadre de la Stratégie de Lisbonne pour 2010, le partage entre femmes et hommes des tâches domestiques et des charges familiales reste inégal. Les politiques de l'Union visent principalement à stimuler une augmentation du taux d'emploi des femmes, en particulier pour prévenir le manque de forces de travail en raison du vieillissement de la population. Malgré un cadre législatif important ayant trait à l'égalité de rémunération entre travailleurs féminins et masculins, l'égalité entre femmes et hommes dans les conditions de travail et la sécurité sociale, ainsi que le congé de maternité et le congé parental, les droits accordés aux travailleurs en matière de conciliation sont parfois relativement restreints. Trois projets législatifs devant le législateur visent à renforcer plusieurs droits, en particulier en ce qui concerne le congé de maternité, le congé parental et les droits de travailleurs indépendants. L'interprétation par la Cour de Justice des droits existants dans ce domaine est bien sûr déterminante. En ce qui concerne les discriminations liées à la grossesse et la maternité, la Cour a assurément renforcé la position des femmes. Mais il est regrettable que sa jurisprudence n'ait en géné-

La présente contribution reprend l'exposé - étoffé et mis à jour - fait le 8 décembre 2009 à l'Académie de droit européen de Trèves dans le cadre du séminaire pour la magistrature intitulé : «Le droit de l'UE sur l'égalité de traitement entre les femmes et les hommes dans la pratique ». Susanne Burri est coordinatrice du réseau d'experts juridiques dans le domaine d'égalité des genres de la Commission européenne.

S. Burri $(\bowtie)$

Faculté de droit, Université d'Utrecht, Achter Sint Pieter 200, 3512 HT Utrecht, The Netherlands e-mail: S.Burri@uu.nl 
ral que peu contribué à réaliser un partage plus équitable des tâches familiales entre femmes et hommes. C'est cet aspect qui mérite le plus d'attention, tant aux niveaux communautaire que national.

Mots clés Égalité hommes et femmes · Discrimination directe · Discrimination indirecte $\cdot$ Congés · Travailleurs indépendants

Abstract This article presents an overview of the objectives of the European Union, the current legal developments at the EU level and the case law of the Court of Justice of the European Union in the area of reconciliation of work, private and family life. While the average female employment rate will probably reach the objective of the Lisbon strategy in 2010, the division of domestic work and family responsibilities between men and women remains unequal. The policies of the EU are mainly aimed at increasing the female employment rate, in particular in order to prevent a shortage in the labour force in the light of an ageing population. Even if an extensive legislative framework exists in relation to equal pay between male and female workers, equality between women and men in working conditions and social security, as well as maternity and parental leave, the rights of workers in relation to reconciliation are sometimes rather limited. Three pending legislative proposals aim to reinforce different rights in particular concerning maternity leave, parental leave and the rights of independent workers. The interpretation by the Court of Justice of existing rights in this area is, of course, decisive. Regarding discrimination in relation to pregnancy and maternity, the Court has certainly reinforced the position of women. It is however regrettable that this case law has, generally speaking, only slightly contributed to realising a more equal division of family responsibilities between women and men. It is in particular this aspect which merits more attention, both at Community and national level.

Keywords Equality between men and women · Direct discrimination · Indirect discrimination $\cdot$ Leave $\cdot$ Independent workers

\section{Introduction}

Faciliter la réconciliation entre la vie professionnelle, vie privée et vie familiale fait partie de la Stratégie de Lisbonne. Une résolution du Conseil datant de 2005 prescrit des lignes directrices aux États membres pour développer cette Stratégie et atteindre les buts définis à Lisbonne : entre autres un taux d'emploi des femmes de $60 \%$ en 2010 en moyenne pour l'UE des 27 États membres. Ce taux était en 2008 en moyenne de $59,1 \%$ (et $72,8 \%$ pour les hommes). ${ }^{1}$ L'objectif est donc presque atteint, mais l'écart entre le taux d'emploi des hommes et des femmes est encore en moyenne de 13,7. Cet écart est plus prononcé dans les pays du Sud de l'Europe (Italie, Espagne, Grèce, Malte par exemple, mais également en Irlande) et moindre dans les pays scandinaves et certains pays de l'Est de l'Europe (Bulgarie, Estonie, Lettonie,

${ }^{1}$ European Commission [5], p. 155. 
Lituanie par exemple). De plus, ce taux d'emploi des femmes est plus bas et l'écart plus grand lorsqu'on compte le taux d'emploi en équivalents à temps plein. En 2007, ces équivalents sont en moyenne pour l'UE des 27 pour les femmes de 48,9\% et pour les hommes de 70,4\%, donc un écart de $21,5 .^{2}$ Cette différence est principalement due au fait que ce sont surtout les femmes qui travaillent à temps partiel. Pour l'UE des 27 , cette moyenne atteint pour les femmes $31,2 \%$ contre $7,7 \%$ pour les hommes. ${ }^{3}$ Une des principales causes de ces différences est le partage encore toujours inégal entre femmes et hommes des tâches domestiques, des charges familiales et de soins à donner aux enfants, personnes âgées ou handicapées. Ainsi, le fait d'avoir un enfant à sa charge de moins de douze ans influence négativement le taux d'emploi des femmes en moyenne de $12,4 \%$, alors que ce taux augmente au contraire pour les hommes de 7,3\% (pour 1'UE des 27). ${ }^{4}$ Les femmes passent en moyenne dans l'UE des 27 États membres 17,7 heures de plus par semaine à remplir des tâches domestiques et familiales que les hommes et travaillent de façon rémunérée 7,4 heures de moins par semaine que les hommes. ${ }^{5}$ Il y a là donc encore toujours d'importants écarts.

La Stratégie de Lisbonne a défini les objectifs en ce qui concerne les taux d'emploi, mais n'a pas indiqué les buts à réaliser en ce qui concerne le partage du travail non rémunéré fait par des personnes privées, comme les tâches domestiques et les responsabilités familiales ou soins à donner. En revanche, en ce qui concerne les gardes d'enfants, le Conseil Européen s'est fixé des objectifs concrets en 2002 à Barcelone. Les États membres devraient offrir des services de gardes pour $90 \%$ des enfants entre trois ans et l'âge de scolarité en 2010. Ce taux devrait être de 33\% pour les enfants de moins de trois ans. Or, un récent rapport de la Commission européenne montre que ces objectifs sont loin d'être atteints et que les parents se voient souvent confrontés à un manque de possibilités de gardes. De plus, celles-ci ne sont souvent disponibles qu'à temps partiel, souvent trop coûteuses et les heures d'ouvertures ne s'articulent pas avec un travail à temps complet ou des heures de travail atypiques. ${ }^{6}$

Les différences de taux d'emploi entre hommes et femmes dépendent entre autres des possibilités institutionnelles offertes dans les différents pays en vue de faciliter la réconciliation. Mais bien sûr des facteurs historiques, socio-économiques, culturels et idéologiques jouent également un rôle important. Le sujet de la réconciliation est étendu et touche des aspects très divers. Il s'agit entre autres des congés (congé de maternité, parental, de paternité, d'adoption, en cas de maladie d'un proche etc.), des différentes possibilités de gardes d'enfants, des actions médico-sociales en relation aux charges de soins à donner aux personnes dépendantes (âgées, handicapées etc.) et des soins à domicile, de l'adaptation du temps de travail, des conditions du travail à temps partiel (et de ses conséquences pour les pensions, la sécurité sociale, le développement de la carrière etc.), de l'adaptation des horaires de travail et certaines

\footnotetext{
${ }^{2}$ European Commission [4], pp. 218-219.

${ }^{3}$ European Commission [6], p. 30.

${ }^{4}$ European Commission [6], p. 31.

${ }^{5}$ European Commission [6], p. 33. Ces chiffres donnent une indication, mais probablement les différences sont plus grandes, car ces statistiques concernent uniquement des personnes ayant un emploi rémunéré.

${ }^{6}$ Rapport de la Commission au Parlement Européen, au Conseil, au Comité Economique et Social Européen et au Comité des Régions. Mise en œuvre des objectifs de Barcelone concernant les structures d'accueil pour les enfants en âge préscolaire, $\operatorname{COM}(2008)$ 638, pp. 3-9.
} 
formes de flexibilité, des interruptions temporaires de la carrière et des conséquences de telles interruptions, de l'approche des cycles de vie, de l'influence des systèmes fiscaux sur la répartition du travail payé et non payé, de l'influence des partenaires sociaux au niveau des secteurs et entreprises en vue de faciliter la réconciliation, etc. ${ }^{7}$

La présente contribution a pour but de rappeler les principaux objectifs communautaires dans le domaine de la réconciliation, d'offrir un aperçu de la législation communautaire en ce domaine et des récents développements législatifs, de discuter de la jurisprudence de la Cour de Justice en particulier en relation aux discriminations directes et indirectes dans le domaine de la réconciliation et de présenter quelques conclusions.

\section{Approches et objectifs communautaires}

La ligne directrice 18 de la Résolution du Conseil sur la Stratégie de Lisbonne stipule qu'il faut favoriser une approche fondée sur le cycle de vie à l'égard du travail. Une telle approche fondée sur le cycle de vie implique selon le Conseil les actions suivantes :

- renouveler les efforts visant à créer des parcours vers l'emploi pour les jeunes et réduire le chômage des jeunes ;

- mener une action déterminée en vue d'accroître l'activité professionnelle des femmes et de réduire les disparités hommes-femmes en matière d'emploi, de chômage et de rémunération ;

- permettre une meilleure conciliation entre vie professionnelle et vie privée et proposer des structures accessibles et abordables de garde d'enfants et d'accueil des autres personnes à charge ;

- soutenir le vieillissement actif, y compris les conditions de travail appropriées, un meilleur état de santé (au travail) et des mesures adéquates favorisant le travail et décourageant la retraite anticipée;

- moderniser les systèmes de protection sociale, y compris les retraites et les soins de santé, pour assurer leur adéquation sociale, leur viabilité financière et leur capacité d'adaptation à l'évolution des besoins, de manière à soutenir l'activité professionnelle et le maintien sur le marché du travail ainsi que la prolongation de la vie professionnelle. ${ }^{8}$

Dans un récent rapport sur l'application de la Stratégie de Lisbonne dans le cadre d'un plan de redressement économique, la Commission européenne signale que peu d'États ont développé une approche globale basée sur les cycles de vie. ${ }^{9}$ Les efforts des Etats membres se concentrent surtout sur le développement des gardes d'enfants.

\footnotetext{
${ }^{7}$ Voir entre autres Plantenga/Remery [11], European Commission [3], European Network of Legal Experts in the field of Gender Equality [7], European Network of Legal Experts in the field of Gender Equality [8]. 82005/600/CE : Décision du Conseil du 12 juillet 2005 relative aux lignes directrices pour les politiques de l'emploi des États membres, JO L 205 du 06/08/2005.

${ }^{9}$ Implementation of the Lisbon Strategy Structural Reforms in the context of the European Economic Recovery Plan-a more detailed overview of progress across the EU in the specific macro- and microeconomic as well as the employment areas, $\operatorname{COM}(2009) 34$, p. 64.
} 
Dans la littérature, une approche de cycle de vie signifie surtout créer plus de possibilités pour pouvoir adapter le temps de travail durant le cycle de vie selon les besoins des salariés en facilitant des transitions entre marché du travail, chômage, formation et éducation et les soins à donner. ${ }^{10}$ En général, les femmes, bien plus souvent que les hommes, effectuent une transition entre un domaine et un autre, en particulier entre l'éducation et les soins à donner et le travail rémunéré. Même si l'approche des cycles de vie est loin d'être développée au niveau communautaire, ou même national, elle me semble intéressante à condition qu'un de ses principaux objectifs soit en effet de faciliter la réconciliation et un partage plus équitable des tâches domestiques et familiales entre femmes et hommes.

Le Conseil européen a approuvé en mars 2006 le Pacte pour l'égalité entre les femmes et les hommes, qui souligne la nécessité de prendre des mesures pour réaliser une meilleure conciliation entre vie professionnelle et vie privée. ${ }^{11}$ La feuille de route pour l'égalité entre femmes et hommes de la Communauté européenne (20062010) mentionne également l'objectif de faciliter la réconciliation, mais ce n'est plus explicitement le cas dans l'agenda social renouvelé. ${ }^{12}$ Là, il est surtout question de la nécessité d'augmenter le taux d'emploi vu les développements démographiques, en particulier le manque de force de travail à cause du vieillissement de la population. ${ }^{13}$ Au niveau communautaire, c'est donc surtout l'augmentation de la participation des femmes sur le marché du travail qui retient l'attention et forme un objectif primordial. Pourtant, plusieurs initiatives législatives intéressantes ont été annoncées dans l'agenda social renouvelé dans le cadre de la lutte contre les discriminations ${ }^{14}$ et ont été prises récemment dans le domaine de la réconciliation au niveau communautaire :

- la proposition pour modifier la directive sur la santé des travailleuses enceintes et le congé de maternité $(92 / 85 / \mathrm{CEE}) ;^{15}$

- la révision de l'accord-cadre des partenaires sociaux sur le congé parental (Directive $96 / 34 / \mathrm{CE}) ;^{16}$

- la proposition de modifier la directive sur l'égalité de traitement des femmes et hommes exerçant une activité indépendante (Directive 86/613/CEE). ${ }^{17}$

\footnotetext{
${ }^{10}$ Voir par exemple Schmid [12] et Schmid [13].

${ }^{11}$ Conclusions de la Présidence, Conseil européen de Bruxelles des 23-24 mars 2006, Annexe II.

${ }^{12}$ Un agenda social renouvelé : opportunités, accès et solidarité dans l'Europe du XXIe siècle, $\operatorname{COM}(2008), 412$.
}

${ }^{13}$ Voir aussi la Résolution du Parlement européen du 21 février 2008 sur l'avenir démographique de l'Europe $(2007 / 2156($ INI)).

${ }^{14} \mathrm{COM}(2008), 412$, p. 16.

${ }^{15}$ Proposition de Directive du Parlement européen et du Conseil portant modification de la directive 92/85/CEE du Conseil concernant la mise en œuvre de mesures visant à promouvoir l'amélioration de la sécurité et de la santé des travailleuses enceintes, accouchées ou allaitantes au travail, COM(2008), 637.

${ }^{16}$ Proposition de Directive du Conseil portant application de l'accord-cadre révisé sur le congé parental conclu par BUSINESSEUROPE, l'UEAPME, le CEEP et la CES et abrogeant la directive 96/34/CE, $\operatorname{COM}(2009), 410$.

${ }^{17}$ Proposition de Directive du Parlement européen et du Conseil concernant l'application du principe de l'égalité de traitement entre hommes et femmes exerçant une activité indépendante et abrogeant la directive 86/613/CEE, COM(2008), 636. 
Récemment, le Conseil de l'UE a réitéré l'importance de traiter les sujets relatifs au genre dans la stratégie post-Lisbonne, entre autres la réconciliation, et a souligné l'importance des gardes d'enfants, des services de soins pour les personnes dépendantes et des mesures pour encourager un partage plus équitable des responsabilités familiales. ${ }^{18}$ Il existe donc maintenant au niveau communautaire un dispositif législatif relativement important sur la réconciliation vie professionnelle, vie familiale et vie privée. De plus, la jurisprudence de la Cour de Justice doit être analysée, étant donné qu'elle doit être appliquée par les États membres, les juges nationaux, les employeurs, les partenaires sociaux, les fonds de pensions, etc.

\section{Législation au niveau communautaire}

\section{1 Égalité de traitement entre femmes et hommes}

Le cadre législatif communautaire sur l'égalité de traitement entre femmes et hommes comprend l'article 157 du Traité sur le Fonctionnement de l'Union Européenne (TFUE), entré en vigueur le $1^{\mathrm{er}}$ décembre 2009 (ex article $141 \mathrm{CE}$ ), de nombreuses directives - entre autres sur la réconciliation - et des recommandations. De plus, beaucoup de documents réfèrent à des aspects concernant la réconciliation. Outre l'article 157 TFUE, qui consacre le principe de l'égalité des rémunérations entre hommes et femmes, les normes communautaires sont ancrées dans différentes directives sur l'égalité entre hommes et femmes, dont la directive relative à l'égalité de traitement entre hommes et femmes en matière d'emploi (76/207/CEE, telle que modifiée par la directive 2002/73/CE), la directive relative à l'égalité de traitement entre hommes et femmes en matière de régime légal de sécurité sociale (79/7/CEE), la directive relative à l'égalité de traitement entre hommes et femmes en matière de régime professionnel de sécurité sociale (86/378/CEE, telle que modifiée par la directive 96/97/CE), la directive relative à l'égalité de traitement entre hommes et femmes exerçant une activité indépendante, y compris une activité agricole (86/613/CEE), la directive relative aux travailleuses enceintes (92/85/CEE), la directive relative au congé parental (96/34/CE), la directive relative à l'égalité entre femmes et hommes dans l'accès à des biens et services et la fourniture de biens et services (2004/113/CE) et, enfin, la directive dite « de refonte » $(2006 / 54 / \mathrm{CE}) .{ }^{19}$ En 1992, une Recommandation concernant la garde d'enfants fut adoptée ${ }^{20}$ et en 2000 , une Résolution sur une participation équilibrée des femmes et hommes à la vie professionnelle et familiale. ${ }^{21}$ De plus, l'article 33 sur la vie familiale et professionnelle de la Charte des droits fondamentaux dispose :

18 Press Release, 2980th Council meeting, Employment, Social Policy, Health and Consumer Affairs, Brussels, 30 November-1 December 2009, http://www.consilium.europa.eu/uedocs/cms_data/docs/ pressdata/en/lsa/111599.pdf.

${ }^{19}$ Voir pour un aperçu général : Burri/Prechal [2].

2092/241/CEE, Recommandation du Conseil, du 31 mars 1992, concernant la garde des enfants, JO L 123 du 08/05/1992.

${ }^{21}$ Résolution du Conseil et des ministres de l'emploi et de la politique sociale, réunis au sein du Conseil du 29 juin 2000 relative à la participation équilibrée des femmes et des hommes à la vie professionnelle et à la vie familiale, JO C 218 du 31/07/2000. 
«1. La protection de la famille est assurée sur le plan juridique, économique et social.

2. Afin de pouvoir concilier vie familiale et vie professionnelle, toute personne a le droit d'être protégée contre tout licenciement pour un motif lié à la maternité, ainsi que le droit à un congé de maternité payé et à un congé parental à la suite de la naissance ou de l'adoption d'un enfant. »

Ainsi, le droit à la réconciliation est reconnu comme étant un droit fondamental et reconnaît l'acquis communautaire en cette matière, mais de plus implique le droit à un congé de maternité payé. ${ }^{22}$

\subsection{Grossesse, accouchement, allaitement et congé de maternité}

La directive 92/85/CEE ${ }^{23}$ concernant la mise en œuvre de mesures visant à promouvoir l'amélioration de la sécurité et de la santé des travailleuses enceintes, accouchées ou allaitantes au travail a pour base légale l'article 118A CEE (article $138 \mathrm{CE}{ }^{24}$ et contient des lignes directrices pour protéger la santé des travailleuses enceintes, accouchées et allaitantes. Il est notamment interdit d'exposer ces travailleuses à des agents chimiques ou biologiques dangereux et le travail de nuit est interdit pendant la grossesse et une certaine période après l'accouchement. Le congé maternité doit comprendre 14 semaines continues au moins, réparties avant et/ou après l'accouchement, dont au moins deux semaines de congé de maternité obligatoire (article 8). Les États membres doivent prendre les mesures nécessaires pour interdire le licenciement des travailleuses pendant la période allant du début de leur grossesse jusqu'au terme du congé de maternité, sauf dans les cas d'exception non liés à leur état (article 10). Cet article a un effet direct (Melgar). ${ }^{25}$ Durant le congé maternité, les droits liés au contrat de travail doivent être assurés et les travailleuses ont droit au maintien de leur rémunération et/ou doivent pouvoir bénéficier d'une prestation adéquate. Une telle prestation est adéquate lorsqu'elle assure des revenus au moins équivalents à ceux que recevrait la travailleuse concernée dans le cas d'une interruption de ses activités pour des raisons liées à son état de santé, dans la limite d'un plafond éventuel déterminé par les législations nationales (article 11 (2)(b) et (3)). Dans l'arrêt McKenna, la Cour de Justice a jugé qu'un régime de congé maladie qui traite de manière identique les travailleurs féminins souffrant d'une maladie liée à une grossesse et les autres travailleurs atteints d'une maladie étrangère à un état de grossesse relève du champ d'application de l'article $141 \mathrm{CE}$ et de la directive 75/117. La Cour a jugé que le montant de ces prestations ne pourrait être minime au point de mettre en cause l'objectif du congé de maternité, qui est de protéger les travailleurs féminins avant et après

\footnotetext{
${ }^{22}$ Voir sur ce sujet : Koukoulis-Spiliotopoulos [9].

${ }^{23}$ Directive 92/85/CEE du Conseil, du 19 octobre 1992, concernant la mise en œuvre de mesures visant à promouvoir l'amélioration de la sécurité et de la santé des travailleuses enceintes, accouchées ou allaitantes au travail (dixième directive particulière au sens de l'article 16 paragraphe 1 de la directive 89/391/CEE), JO L 348 du 28/11/1992.

${ }^{24}$ Article 154 TFUE.

${ }^{25}$ Arrêt du 4 octobre 2001, Maria Luisa Jiménez Melgar/Ayuntamiento de Los Barrios (C-438/99, Rec. P. I-06915, point 34).
} 
l'accouchement. Un état pathologique lié à la grossesse ou à l'accouchement, apparu après le congé de maternité, peut entraîner une réduction de la rémunération dans les mêmes conditions qu'une autre maladie. ${ }^{26}$

Le projet de loi en vue de modifier les propositions de la directive relative aux travailleuses enceintes $(92 / 85 / \mathrm{CEE})$ concerne principalement la durée du congé de maternité, la protection contre le licenciement et les droits des travailleuses enceintes et qui ont récemment donné naissance à un enfant. ${ }^{27}$ La durée du congé maternité passerait de 14 à 18 semaines (article 8), dont six semaines au moins devraient être prises après la naissance. Les travailleuses auraient le droit de choisir librement la période - avant ou après l'accouchement - à laquelle elles prennent la partie non obligatoire de leur congé maternité et ne seraient donc plus obligées de prendre une partie déterminée du congé avant la naissance, comme c'est actuellement le cas dans certains États membres. Toute période d'absence jusqu'à quatre semaines avant la naissance en cas de maladie ou de complications liées à la grossesse ne pourrait être prise en considération pour écourter le congé de maternité. L'article 10 interdirait tout préparatif pour un licenciement durant le congé de maternité qui ne serait pas dû à des circonstances exceptionnelles. De plus, si une femme est licenciée dans les six mois suivant le congé maternité, elle aurait le droit de demander les motifs du licenciement par écrit. La rémunération serait équivalente au salaire complet reçu au début du congé maternité. Les États membres auraient néanmoins le droit de déterminer un plafond dans la législation nationale, mais ce plafond ne pourrait être inférieur au montant qu'une travailleuse recevrait en cas d'absence pour raison de santé (article 11(1)(a)). La travailleuse aurait le droit pendant ou après le congé maternité de demander à son employeur de modifier ses rythme et horaire de travail. L'employeur serait tenu d'examiner une telle requête en tenant compte des besoins des deux parties (article 11(5)). Le projet de loi contient également des clauses sur la charge de la preuve, la protection contre les rétorsions etc. Ce projet de la Commission n'est pas encore adopté, on attend la décision du Parlement européen.

\subsection{Congé parental et absence de travail pour force majeure}

La directive 96/34/CE 28 oblige les États membres à mettre en œuvre l'accord-cadre des partenaires sociaux sur le congé parental. Cet accord s'applique à tous les travailleurs, hommes et femmes, ayant un contrat ou une relation de travail définie par la législation, les conventions collectives ou pratiques en vigueur dans chaque État membre (clause 1(2)). Ces travailleurs ont un droit individuel à un congé parental en raison de la naissance ou de l'adoption d'un enfant, pour pouvoir s'occuper de cet enfant pendant au moins trois mois jusqu'à un âge déterminé pouvant aller jusqu'à huit ans, à définir par les États membres et/ou les partenaires sociaux (clause 2(1)). Ce congé devrait, en principe, être accordé de manière non transférable (clause 2(2)).

\footnotetext{
${ }^{26}$ Arrêt du 8 septembre 2005, North Western Health Board/Margaret McKenna (C-191/03, Rec. P. I-07631).

${ }^{27} \mathrm{COM}(2008) 637$.

${ }^{28}$ Directive 96/34/CE du Conseil du 3 juin 1996 concernant l'accord-cadre sur le congé parental conclu par l'UNICE, le CEEP et la CES, JO L 145 du 19/06/1996.
} 
Ce congé peut être accordé à temps plein, à temps partiel, de manière fragmentée, ou sous forme d'un crédit-temps et il peut être subordonné à une période de travail et/ou une période d'ancienneté qui ne peut dépasser un an (clause 2(3)). Une protection contre le licenciement est prévue et à l'issue du congé parental, le travailleur a le droit de retrouver son poste de travail ou, en cas d'impossibilité, un travail équivalent ou similaire conforme à son contrat ou à sa relation de travail (clause 2(5)). La clause 2(6) dispose que les droits acquis ou en cours d'acquisition par le travailleur à la date du début du congé parental sont maintenus dans leur état jusqu'à la fin du congé parental. À l'issue du congé parental, ces droits, y compris les changements provenant de la législation, de conventions collectives ou de la pratique nationale, s'appliquent. La Cour de Justice a récemment jugé que la clause 2(6) peut être invoquée par des particuliers devant leur juridiction nationale. Par contre, ce n'est pas le cas de la clause 2(8) qui concerne les questions de sécurité sociale liées au congé parental et qui n'impose aux États membres aucune obligation de prévoir, pendant la durée du congé parental, la continuité de la perception, par le travailleur, de prestations de sécurité sociale et ne définit pas de droits au profit des travailleurs (Gómez-Limón). ${ }^{29}$ Dans un arrêt récent, la Cour a jugé que la clause 2, points 6 et 7, de l'accord-cadre sur le congé parental doit être interprétée en ce sens qu'elle s'oppose à ce que, en cas de résiliation unilatérale par l'employeur, sans motif grave ou sans respecter le délai légal de préavis, du contrat de travail d'un travailleur engagé à durée indéterminée et à temps plein alors que ce dernier bénéficie d'un congé parental à temps partiel, l'indemnité à verser à ce travailleur soit déterminée sur la base de la rémunération réduite qu'il perçoit lorsque le licenciement intervient. Dans ce cas, le travailleur ayant un contrat à temps complet et bénéficiant d'un congé parental à temps partiel et le travailleur à temps plein ne se trouvent pas dans une situation différente par rapport au contrat de travail initial qui les lie à l'employeur (Meerts). ${ }^{30}$

Les travailleurs ont le droit de s'absenter de leur travail pour cause de force majeure liée à des raisons familiales urgentes en cas de maladie ou d'accident rendant indispensable la présence immédiate du travailleur (clause 3).

En juin 2009, cet accord-cadre a été révisé par les partenaires sociaux européens (BUSINESSEUROPE, l'UEAPME, le CEEP et la CES). ${ }^{31}$ L'accord révisé prolonge d'un mois le congé parental auquel ont droit les travailleurs des deux sexes pour cause de naissance d'un enfant ou d'adoption. Le congé parental sera donc de quatre mois à prendre jusqu'à un âge maximum de l'enfant de huit ans. Le congé parental est un droit individuel qui devrait en principe ne pas être transféré d'un parent à l'autre. En outre, l'accord prévoit qu'au moins un des quatre mois ne peut être transféré en aucune circonstance et ce mois est perdu si seul un des parents prend le congé parental. L'accord s'appliquera à tous les types de contrat et de relation de travail, notamment le travail à temps partiel, le travail à durée déterminée et le travail intérimaire. Les

\footnotetext{
${ }^{29}$ Arrêt du 16 juillet 2009 Evangelina Gómez-Limón Sánchez-Camacho / Instituto Nacional de la Seguridad Social (INSS), Tesorería General de la Seguridad Social (TGSS), Alcampo SA (C-537/07, pas encore publié).

${ }^{30}$ Arrêt du 22 octobre 2009, Christel Meerts/Proost NV (C-116/08, pas encore publié).

31 Press Releases Rapid, 18 juin 2009, http://europa.eu/rapid/pressReleasesAction.do?reference=IP/09/ $948 \&$ format $=$ HTML\&aged $=0$ \&language $=F R \&$ guiLanguage $=$ en .
} 
États membres doivent prendre des mesures nécessaires pour protéger les travailleurs contre un traitement défavorable ou le licenciement pour demande ou prise de congé parental. Les travailleurs peuvent à leur retour du congé parental demander l'aménagement de leur horaire ou de leur rythme de travail pendant une période donnée. La Commission européenne a publié un projet de directive pour la mise en œuvre de cet accord, qui sera probablement adopté. ${ }^{32}$ Le Conseil de l'UE a conclu un accord politique concernant ce projet le 30 novembre 2009 et cette directive devrait entrer en vigueur début $2012 .{ }^{33}$

\subsection{Travailleurs indépendants}

Un autre projet de directive a pour but de modifier la directive relative à l'application du principe de l'égalité de traitement entre hommes et femmes aux travailleurs indépendants et à leurs conjoints. ${ }^{34}$ Elle abrogera la directive $86 / 613 / \mathrm{CEE}^{35}$ et modernisera les dispositions relatives aux travailleurs indépendants. En ce qui concerne la réconciliation, l'article 7, paragraphe 1 du projet dispose que les femmes exerçant une activité indépendante et les conjointes aidantes devraient pouvoir bénéficier, à leur demande, du congé maternité prévu par la directive 92/85/CEE. Ce congé maternité devrait être rémunéré à un taux au moins équivalent aux indemnités reçues en cas de maladie, sous réserve d'un éventuel plafond fixé dans la législation nationale. Si la personne en question ne bénéficie pas d'une indemnité de maladie, le paiement devrait être équivalent à toute allocation appropriée existant au niveau national. Il serait éventuellement possible de bénéficier de services de remplacement temporaire, au lieu d'une allocation financière. Cela devrait permettre aux femmes exerçant une activité indépendante de prendre leur congé de maternité et de maintenir leur activité professionnelle grâce à un remplacement temporaire. Ce projet reflète les efforts faits par la Commission pour harmoniser les dispositions de différentes directives : ainsi par exemple les concepts de discrimination directe, de discrimination indirecte, de harcèlement sont définis de façon semblable. Les dispositions horizontales concernant notamment la charge de la preuve, les sanctions, le rôle des partenaires sociaux sont également modelées sur les autres directives communautaires sur l'égalité de traitement. Sur ce dossier également, le Conseil a conclu un accord politique le 30 novembre 2009.36

\footnotetext{
${ }^{32} \operatorname{COM}(2009) 410$.

33 Press Release, 2980th Council meeting, Employment, Social Policy, Health and Consumer Affairs, Brussels, 30 November-1 December 2009, http://www.consilium.europa.eu/uedocs/cms_data/docs/ pressdata/en/lsa/111599.pdf.

${ }^{34} \mathrm{COM}(2008), 636$.

${ }^{35}$ Directive 86/613/CEE du Conseil du 11 décembre 1986 sur l'application du principe de l'égalité de traitement entre hommes et femmes exerçant une activité indépendante, y compris une activité agricole, ainsi que sur la protection de la maternité, JO L 359 du 19/12/1986.

36 Press Release, 2980th Council meeting, Employment, Social Policy, Health and Consumer Affairs, Brussels, 30 November-1 December 2009, http://www.consilium.europa.eu/uedocs/cms_data/docs/ pressdata/en/lsa/111599.pdf.
} 


\section{Discriminations directes et indirectes}

La Cour de Justice a interprété, à plusieurs reprises, les dispositions relatives à l'égalité de traitement entre femmes et hommes et les directives concernant la grossesse, la maternité et le congé parental. En ce qui concerne la grossesse et la maternité, la Cour a estimé en 1984 dans l'arrêt Hofmann que dans le cadre de la directive 76/207 il s'agit «d'une part d'assurer la protection biologique de la femme au cours de sa grossesse et à la suite de celle-ci, jusqu'à un moment où ses fonctions physiologiques et psychiques sont normalisées à la suite de l'accouchement et d'autre part, la protection des rapports particuliers entre la femme et son enfant au cours de la période qui fait suite à la grossesse et l'accouchement, en évitant que ces rapports soient troublés par le cumul des charges résultant de l'exercice simultané d'une activité professionnelle ». ${ }^{37}$ Cette approche de la Cour a été critiquée pour son insistance sur la protection de la femme, en particulier en relation à l'enfant, qui risque d'entraver un réel partage des responsabilités familiales entre femmes et hommes. ${ }^{38}$ Treize ans plus tard la Cour a entre autres constaté que : «La politique communautaire (...) consiste à encourager et si possible à adapter les conditions du travail aux charges de famille. La protection de la femme dans la vie familiale ainsi que dans le déroulement de son activité professionnelle est, tout comme celle de l'homme, un principe qui est largement considéré dans les ordres juridiques des États membres comme étant le corollaire naturel de l'égalité entre hommes et femmes et reconnu par le droit communautaire » (Gerster et Hill). ${ }^{39}$ Même si la Cour de Justice insiste encore sur la protection de la femme (et de l'homme) dans ce domaine, le fait qu'elle souligne que ce principe est le corollaire naturel de l'égalité entre hommes et femmes offre des possibilités accrues d'appliquer ce principe pour traiter des problèmes dans ce domaine, même lorsqu'il n'existe pas de droits spécifiques dans un cas particulier. Il est bien sûr préférable pour les salariés de pouvoir invoquer des droits distinctifs, comme ceux décrits ci-dessus, qui sont souvent clairement définis. Mais la jurisprudence de la Cour offre également des exemples comment des discriminations directes et indirectes fondées sur le sexe peuvent être combattues en appliquant les dispositions communautaires sur l'égalité de traitement entre femmes et hommes. La Cour a en outre précisé que la directive 76/207 vise à déboucher sur une égalité substantielle et non formelle (Thibault). ${ }^{40}$

\subsection{Discriminations directes}

Dans la plupart des directives mentionnées sous le paragraphe 3.1, les définitions des discriminations directes et indirectes sont semblables. La discrimination directe est

\footnotetext{
${ }^{37}$ Arrêt du 12 juillet 1984, Ulrich Hofmann/ Barmer Ersatzkasse (184/83, Rec. P. 3047, point 25).

${ }^{38}$ Voir par exemple McGlynn [10].

${ }^{39}$ Arrêt du 2 octobre 1997, Hellen Gerster/Freistaat Bayern, (C-1/95, Rec. P. I-05253, point 38) et arrêt du 17 juin 1998, Kathleen Hill et Ann Stapleton/The Revenue Commissioners et Department of Finance, (C-243/95, Rec. P. I-03739, point 42).

${ }^{40}$ Arrêt du 30 avril 1998, Caisse nationale d'assurance vieillesse des travailleurs salariés (CNAVTS)/ Evelyne Thibault (C-136/95, Rec. P. I-2011, point 26).
} 
définie comme « la situation dans laquelle une personne est traitée de manière moins favorable en raison de son sexe qu'une autre ne l'est, ne l'a été ou ne le serait dans une situation comparable ». ${ }^{41}$ Mais une comparaison n'est pas exigée lorsqu'une mesure désavantage une femme enceinte. La Cour a en effet estimé que le refus d'embaucher une femme parce qu'elle est enceinte constitue une discrimination directe fondée sur le sexe, laquelle est interdite. Le fait qu'il n'y ait pas de candidats masculins est dénué de pertinence si la décision de ne pas engager la candidate est liée à sa grossesse (Dekker). ${ }^{42}$ La Cour a également établi que, même si la grossesse n'est aucunement assimilable à un état pathologique, il n'en reste pas moins qu'elle correspond à une période au cours de laquelle des troubles et des complications peuvent survenir et contraindre une femme à une surveillance médicale stricte, voire, dans certains cas, à un repos absolu durant toute la durée ou une partie de la grossesse. Ces troubles et complications, qui peuvent entraîner une incapacité de travail, relèvent des risques inhérents à l'état de grossesse, et un traitement moins favorable lié à ce motif équivaut également à une discrimination directe. La Cour a ainsi jugé que le licenciement d'un travailleur féminin qui intervient au cours de la grossesse pour cause d'absences dues à l'incapacité de travail découlant de la grossesse est lié à la survenance des risques inhérents à la grossesse et doit donc être regardé comme fondé essentiellement sur le fait de la grossesse. Un tel licenciement ne peut concerner que les femmes et constitue dès lors une discrimination directe fondée sur le sexe (Brown). ${ }^{43}$ Cette protection contre le licenciement pour cause de grossesse s'applique également à une travailleuse engagée sur contrat à durée déterminée (CDD), même au cas où elle n'a pas informé l'employeur de son état au moment de la conclusion du CDD et qu'elle n'est pas en mesure de travailler pendant un grande partie de la durée du CDD (Tele Danmark et Melgar). ${ }^{44}$ Le législateur européen a clairement précisé dans la directive « refonte » que tout traitement moins favorable d'une femme lié à la grossesse ou au congé de maternité est visé par l'interdiction de discriminer (article 2(2)(c)).

Enfin, en ce qui concerne la distinction entre la discrimination directe et la discrimination indirecte, il convient de souligner que la discrimination directe est totalement interdite, sauf dans les strictes limites des dérogations écrites et spécifiques prévues. Ces exceptions diffèrent d'une directive à l'autre. Aucune exception n'est permise au principe d'égalité de rémunérations entre hommes et femmes, sauf s'il s'agit de mesures pour assurer concrètement une pleine égalité entre hommes et femmes dans la vie professionnelle. L'article 157(4) TFUE dispose que « le principe de l'égalité de traitement n'empêche pas un État membre de maintenir ou d'adopter des mesures prévoyant des avantages spécifiques destinés à faciliter l'exercice d'une activité professionnelle par le sexe sous-représenté ou à prévenir ou compenser des

\footnotetext{
${ }^{41}$ Directive $2006 / 54 / \mathrm{CE}$, article 2 (1) (a).

${ }^{42}$ Arrêt du 8 novembre 1990, Elisabeth Johanna Pacifica Dekker/Stichting Vormingscentrum (177/88, Rec. P. I-3941, point 17).

${ }^{43}$ Arrêt du 30 juin 1998, Mary Brown/Rentokil Ltd (C-394/96, Rec. P. I-4185, points 22 et 28).

${ }^{44}$ Arrêt du 4 octobre 2001, Tele Danmark A/S/Handels- og Kontorfunktionærernes Forbund i Danmark (HK), (C-109/00. Rec. P. I-06993) et arrêt du 4 octobre 2001, Maria Luisa Jiménez Melgar/Ayuntamiento de Los Barrios, (C-438/99, Rec. P.I-06915). Dans ces cas, l'article 10 de la Directive 95/82 était également applicable.
} 
désavantages dans la carrière professionnelle ». Mais cette exception est interprétée de façon stricte par la Cour (Abrahamsson). ${ }^{45}$ Dans certains pays, les politiques de réconciliation visent en particulier les femmes ou les jeunes mamans et peuvent éventuellement former de discriminations directes interdites par le droit communautaire. Par exemple, dans un arrêt de 1988, Commission/France, il s'agissait de droits spécifiques octroyés aux femmes seulement dans des conventions collectives concernant par exemple une réduction de la durée du travail, l'avancement de l'âge de la retraite, l'obtention de congés pour enfants malades, l'octroi de jours supplémentaires de congé annuel par enfant, l'octroi d'heures de congé à l'occasion de la fête des mères, l'octroi de bonifications pour le calcul de la retraite etc. La Cour a jugé que certains de ces droits étaient contraires au principe d'égalité de traitement s'appliquant aux conditions de travail. ${ }^{46}$ Plus récemment, l'affaire Griesmar concernait une bonification d'ancienneté pour le calcul de la pension de retraite réservée aux fonctionnaires féminins ayant eu des enfants. Les fonctionnaires masculins, même s'ils pouvaient prouver avoir assumé effectivement l'éducation des enfants, en étaient exclus. La Cour de Justice a jugé cette bonification contraire au principe d'égalité de rémunération. Selon la Cour, cette bonification ne constituait pas une mesure d'action positive, car « la mesure en cause se borne à accorder aux fonctionnaires féminins ayant la qualité de mère une bonification d'ancienneté au moment de leur départ à la retraite, sans porter remède aux problèmes qu'ils peuvent rencontrer durant leur carrière professionnelle. » ${ }^{47}$ Il est difficile de sortir du dilemme entre d'une part tenir compte des charges et responsabilités réparties inégalement entre femmes et hommes, surtout dans le passé, mais encore à présent et d'autre part, de respecter le principe d'égalité de traitement et de favoriser une répartition plus égale des responsabilités domestiques et familiales. En général, il s'agit de formuler des droits de façon neutre, dont peuvent profiter autant les femmes que les hommes, sans qu'un des deux sexes ne soit désavantagé. Le résultat doit correspondre à une égalité substantielle.

\subsection{Discriminations indirectes}

La définition d'une discrimination indirecte est également semblable dans les directives mentionnées au paragraphe 3.1. Une discrimination indirecte est « la situation dans laquelle une disposition, un critère ou une pratique apparemment neutre désavantagerait particulièrement des personnes d'un sexe par rapport à des personnes de l'autre sexe, à moins que cette disposition, ce critère ou cette pratique ne soit objectivement justifié par un but légitime et que les moyens pour parvenir à ce but soient appropriés et nécessaires. » ${ }^{48}$

\footnotetext{
${ }^{45}$ Voir article 141(4) CE, arrêt du 6 juillet 2000, Katarina Abrahamsson et Leif Anderson/Elisabet Fogelqvist (C-407/98, Rec. P. I-5539).

${ }^{46}$ Article 2, paragraphe 3 et paragraphe 4 de la Directive 76/207/CEE, arrêt du 25 octobre 1988, Commission/République française, (312/86, Rec. P. 6315).

${ }^{47}$ Article 119 CEE, maintenant article 157 TFCE, arrêt du 29 novembre 2001, Joseph Griesmar/Ministre de l'Economie, des Finances et de l'Industrie et Ministre de la Fonction publique, de la Réforme de l'Etat et de la Décentralisation, (C-366/99, Rec. P. I-09383, point 65).

${ }^{48}$ Directive 2006/54/CE, article 2 (1) (b).
} 
Beaucoup de discriminations indirectes ont trait au travail à temps partiel. Dans ce domaine, il existe une jurisprudence de la Cour très développée. ${ }^{49}$ Dans l'arrêt Bilka la Cour de Justice a estimé que l'article 119 CEE sur l'égalité de rémunération des travailleurs féminins et masculins est violé par une entreprise qui exclut les employés à temps partiel du régime de pensions d'entreprise lorsque cette mesure frappe un nombre beaucoup plus élevé de femmes que d'hommes, à moins que l'entreprise démontre que cette exclusion s'explique par des facteurs objectivement justifiés et étrangers à toute discrimination sur le sexe. Ceci peut être le cas si l'objectif de la mesure répond à un besoin réel de l'entreprise et les moyens choisis pour atteindre cet objectif sont à la fois adéquats et nécessaires. ${ }^{50} \mathrm{Il}$ découle de la jurisprudence de la Cour de Justice que ne pas tenir compte des charges domestiques et familiales inégales peut également former une discrimination indirecte fondée sur le sexe. Ainsi la Cour de Justice a reconnu par exemple dans l'affaire Danfoss en 1989 déjà, que si le critère de flexibilité est entendu comme recouvrant «l' adaptabilité du travailleur à des horaires et des lieux de travail variables, le critère de la flexibilité peut également jouer au détriment des travailleurs féminins, qui, en raison des tâches ménagères et familiales dont elles ont souvent la responsabilité, peuvent, moins aisément que les travailleurs masculins, organiser leur temps de travail de façon souple. » L'employeur peut justifier le recours au critère de la flexibilité s'il est entendu comme visant à l'adaptabilité à des horaires et des lieux de travail variables, en démontrant que cette adaptabilité revêt de l'importance pour l'exécution des tâches spécifiques qui sont confiées au travailleur. ${ }^{51}$ Ainsi, la Cour reconnaît que l'application du critère de flexibilité ne peut être justifiée que pour certaines tâches spécifiques. Certaines formes de flexibilité peuvent rendre la réconciliation plus difficile au lieu de la faciliter.

Mais la Cour de Justice n'a pas toujours tenu compte de telles responsabilités en dehors du travail dans sa jurisprudence. C'est par exemple le cas de la jurisprudence concernant des majorations dues en cas d'heures supplémentaires des travailleurs à temps partiel. Les salariés travaillant à temps partiel ont souvent des responsabilités en dehors des heures de travail agréées, par exemple pour raisons familiales. Des heures supplémentaires impliquent alors pour eux autant d'inconvénients que pour les travailleurs à temps complet. La Cour a cependant considéré dans l'affaire $\mathrm{Hel}$ mig que le principe d'égalité des rémunérations entre hommes et femmes ne s'oppose pas «à ce qu'une convention collective ne prévoie le paiement de majorations pour heures supplémentaires, aussi bien pour les travailleurs à temps partiel que pour les travailleurs à temps plein, uniquement en cas de dépassement de la durée normale de travail qu'elle fixe et non pas en cas de dépassement de la durée prévue par les contrats individuels de travail. » Selon la Cour de telles dispositions ne créent pas de différence de traitement entre les travailleurs à temps partiel et ceux à temps

\footnotetext{
${ }^{49}$ Voir par exemple Traversa [15].

${ }^{50}$ Arrêt du 13 mai 1986, Bilka-Kaufhaus GmbH/Karin Weber von Hartz Affaire (170/84, Rec. P. 01607, point 36).

${ }^{51}$ Arrêt du 17 octobre 1989, Handels- og Kontorfunktionærernes Forbund I Danmark/Dansk Arbejdsgiverforening, agissant pour Danfoss, (C-109/88, Rec. P. 3199, point 25).
} 
plein, puisque les travailleurs à temps partiel reçoivent, à parité d'heures effectuées, la même rémunération globale que celle perçue par les travailleurs à temps plein. ${ }^{52} \mathrm{Ce}$ jugement a été vivement critiqué, mais la Cour a suivi la même approche dans deux arrêts plus récents. ${ }^{53} \mathrm{La}$ Cour a adopté une approche similaire en ce qui concerne l'acquisition de prestations de sécurité sociale (en ce cas une pension d'invalidité permanente) durant le congé parental. Dans la récente affaire Goméz-Limon, où il s'agit de savoir s'il y a discrimination indirecte fondée sur le sexe, la Cour reconnait que «... afin de se consacrer à l'éducation des enfants, les femmes optent bien plus souvent que les hommes pour des périodes de réduction de l'horaire de travail assorties d'une réduction proportionnelle du salaire ayant pour conséquence une diminution des droits de sécurité sociale dérivés de la relation de travail. » Mais elle poursuit : «Il ressort toutefois d'une jurisprudence également constante qu'une discrimination consiste dans l'application de règles différentes à des situations comparables ou dans l'application de la même règle à des situations différentes. » «Or, le travailleur, qui bénéficie du congé parental, (...) en exerçant, comme dans l'affaire au principal, une activité à temps partiel, se trouve dans une situation spécifique, qui ne peut être assimilée à celle d'un homme ou d'une femme qui travaille à temps plein. " ${ }^{54}$ Ainsi le problème de la comparabilité se pose ici de telle façon que les possibilités d'appliquer le concept de discriminations indirectes dans le domaine de la sécurité sociale s'avèrent plus restreintes qu'en ce qui concerne les rémunérations, l'accès à l'emploi, les conditions de travail et le licenciement, en particulier en ce qui concerne les discriminations indirectes en relation au travail à temps partiel. Ce concept peut - même s'il a certainement des limites - néanmoins avoir un impact important dans le sens où des situations qui désavantagent en particulier les femmes vues leurs responsabilités familiales peuvent tomber sous l'application du droit communautaire et sont interdites si elles ne sont pas objectivement justifiées. ${ }^{55}$

\section{Quelques conclusions}

Le droit communautaire couvre maintenant un domaine étendu et les références à la réconciliation entre vie professionnelle et vie privée sont nombreuses. Plusieurs directives obligent les États membres à mettre en œuvre des droits spécifiques en relation avec la grossesse, la maternité, le soin des enfants. Il y a lieu de souligner l'importance de l'interdiction des discriminations directes fondées sur le sexe et le rôle positif que la Cour de Justice a joué dans ce domaine. La protection des femmes en relation avec la grossesse et la maternité dans l'accès à l'emploi, les conditions

\footnotetext{
${ }^{52}$ Arrêt du 15 décembre 1994, Stadt Lengerich/Angelika Helmig et al. (affaires jointes C-399/92, C-409/92, C-425/92, C-34/93, C-50/93 et C-78/93, Rec. 1994 P. I-05727, points 26 à 31). Voir aussi Burri [1].

${ }^{53}$ Arrêt du 27 mai 2004, Edeltraud Elsner-Lakeberg/Land Nordrhein-Westfalen, (C-285/02, Rec. P. I-5861) et arrêt du 6 décembre 2007, Ursula Voß/Land Berlin (C-300/06, Rec. P. I-10573).

${ }^{54}$ Arrêt du 16 juillet 2009 Evangelina Gómez-Limón Sánchez-Camacho / Instituto Nacional de la Seguridad Social (INSS), Tesorería General de la Seguridad Social (TGSS), Alcampo SA, (C-537/07, points 55 à 57 , pas encore publié).

${ }^{55}$ Voir Tobler [14].
} 
d'emploi et le licenciement est certainement améliorée. L'application du concept de discrimination indirecte fondée sur le sexe a par contre moins d'impact, surtout dans le domaine de la sécurité sociale. Le problème de la comparabilité dans l'approche de la Cour restreint dans certains cas malheureusement les possibilités d'appliquer ce concept.

Si le projet de modifier la directive 92/85/CEE est adopté, les droits des travailleuses liés à la grossesse et la maternité seraient renforcés, en particulier en ce qui concerne le congé de maternité payé, surtout dans les pays qui réaliseraient un congé payé à $100 \%$ alors que ce n'est pas encore le cas maintenant. Néanmoins, les États membres ont la possibilité de définir un plafond, pourvu qu'il ne soit pas inférieur à une allocation reçue en cas de maladie. Les droits en relation avec la grossesse et la maternité des travailleuses indépendantes sont bien moins définis. C'est également le cas des droits visant à faciliter la réconciliation de la vie professionnelle, vie privée et vie familiale, non seulement des femmes, mais également des hommes. Il est bien sûr important de renforcer les droits des femmes en ce qui concerne en particulier les aspects financiers des congés de maternité, mais un congé de maternité relativement long ne contribue en soi pas à un partage plus équitable des tâches domestiques et familiales. Il est donc crucial que des droits soient attribués aux deux parents (et aux parents adoptifs) et dans ce sens, l'accord révisé sur le congé parental est un pas - bien que modeste - dans la bonne direction. Ceci est d'autant plus important vu les différences de salaires et la ségrégation horizontale entre femmes et hommes, qui expliquent en partie pourquoi les femmes bien plus souvent que les hommes prennent des congés. Si les conditions de ces congés sont défavorables, parce qu'ils sont par exemple non payés ou ont d'importantes conséquences pour les pensions et/ou la sécurité sociale, ce sont le plus souvent les femmes qui en souffrent disproportionnellement.

Nous sommes encore loin d'un partage équitable des tâches domestiques et des responsabilités familiales entre femmes et hommes en Europe. Un tel partage présuppose des droits neutres pour femmes et hommes et non pas une approche qui renforce la protection des femmes plus que nécessaire. Dans ce sens il serait préférable de réaliser un congé parental à de meilleures conditions au niveau communautaire. Pour certains pays, une amélioration des normes minimales au niveau communautaire signifie également une meilleure législation au niveau national. Les normes communautaires sont donc ici les bienvenues, bien que, par les différentes bases légales, il soit difficile de développer une approche intégrée entre par exemple différentes formes de congé (congé maternité, congé parental, congé paternité et congé pour soins à donner par exemple). Des politiques basées sur les cycles de vie sont également encore peu développées.

Les approches au niveau national dans le domaine de la réconciliation sont très diverses et méritent certainement notre attention. Il semble primordial de développer, autant au niveau communautaire qu' au niveau national, non seulement des politiques visant à réaliser un taux d'emploi des femmes plus égal à celui des hommes, mais également des approches ayant pour but de faciliter un partage plus équitable des tâches domestiques et des responsabilités familiales.

Open Access This article is distributed under the terms of the Creative Commons Attribution Noncommercial License which permits any noncommercial use, distribution, and reproduction in any medium, provided the original author(s) and source are credited. 


\section{Bibliographie}

1. Burri, S. : Un regard critique sur l'approche du genre en droit du travail communautaire. In : Auvergnon, P. (réd.) : Genre et droit social, pp. 53-71. Presses universitaires de Bordeaux, Bordeaux (2008)

2. Burri, S., Prechal, S. : L'égalité des genres dans le droit de l'UE, Luxembourg, Office des Publications Officielles des Communautés européennes (2008), sur http://bookshop.europa.eu/eubookshop/ publicationDetails.action?pubuid=640631\&offset=3; http://ec.europa.eu/social $/ \mathrm{main} . j$ sp? $\mathrm{catId}=641 \&$ langId=en

3. European Commission : Reconciliation of professional and private life: exchange of good practices. Office for Official Publications of the European Communities, Luxembourg (2006)

4. European Commission : Employment in Europe 2008, Office for Official Publications of the European Communities, Luxembourg, October 2008

5. European Commission : Employment in Europe 2009, Office for Official Publications of the European Communities, Luxembourg, October 2009

6. European Commission : Report on Equality between Women and Men 2009, Office for Official Publications of the European Communities, Luxembourg (2009)

7. European Network of Legal Experts in the field of Gender Equality : Report on Pregnancy, Maternity, Parental and Paternity Rights, European Commission (2007), sur http://ec.europa.eu/social/main.jsp? catId=641\&langId=en

8. European Network of Legal Experts in the field of Gender Equality : Legal Approaches to Some Aspects of the Reconciliation of Work, Private and Family Life in Thirty European Countries. European Commission (2008), sur http://ec.europa.eu/social/main.jsp?catId=641\&langId=en

9. Koukoulis-Spiliotopoulos, S. : Le Traité de Lisbonne et la Charte des droits fondamentaux : maintenir et développer l'acquis en matière d'égalité des genres. Revue du droit européen de l'égalité des genres, $n^{\mathrm{o}} 1$, pp. 16-25 (2008), sur http://ec.europa.eu/social/main.jsp?catId=641\&langId=en

10. McGlynn, C. : Ideologies of motherhood in European community sex equality law. Eur. Law J. 6, 29-44 (2000)

11. Plantenga, J., Remery, C. : EU Expert Group on Gender, Social Inclusion and Employment, European Commission, Reconciliation of work and private life: a comparative review of thirty European countries, Office for Official Publications of the European Communities, Luxembourg (2005)

12. Schmid, G. : Transitional labour markets and the European social model: towards a new employment compact. In : Schmid, G., Grazier, B. (réds.) The Dynamics of Full Employment. Social Integration Through Transitional Labour Markets. Edward Elgar, Cheltenham (2002)

13. Schmid, G. : Employment insurance for managing critical transitions during the life cycle. In : Auer, P., Graxier, B. (réds.) The Future of Work, Employment and Social Protection, International Institute for Labour Studies, Geneva (2002)

14. Tobler, C. : European network of legal experts in the non-discrimination field, Limits and potential of the concept of indirect discrimination, European Commission (2008), sur http://ec.europa.eu/social/ main.jsp?catId=615\&langId=fr

15. Traversa, E. : The protection of part-time workers in the case law of the court of justice of the European communities. Int. J. Comp. Labour Law Ind. Relat. 19(2), 219-241 (2003) 